\title{
Contaduría y Administración
}

Indexada en:

- Conacyt (Índice de Revistas Mexicanas de Investigación Científica y Tecnológica del Consejo Nacional de Ciencia y Tecnología)

- Scielo Citation Index -Journal Master List (Thomson Reuters)-

- EconLit (The American Economic Association's electronic bibliography)

- Repec (Research Papers in Economics)

- Redalyc (Red de Revistas Científicas de América Latina y el Caribe, España y Portugal en Ciencias Sociales y Humanidades)

- Dialnet

- Scielo (Scientific Electronic Library Online, México)

- Doaj (Directory of Open Access Journals)

- Catálogo Latindex (Sistema Regional de Información en Línea para Revistas Científicas de América Latina, del Caribe, España y Portugal)

- Clase (Citas Latinoamericanas en Ciencias Sociales y Humanidades)

- Iresie (Índice de Revistas de Educación Superior e Investigación Educativa)

- Catálogo de Revistas Científicas y Arbitradas de la UNAM

Versión electrónica (en texto completo) disponible en:

http://contaduriayadministracionunam.mx 



\section{Editorial}

Aprovechando la ocasión para saludar a nuestros amables lectores, manifestamos una vez más nuestra voluntad para que los esfuerzos editoriales que llevamos a cabo redunden en la producción de una revista de contenidos de alta calidad. Naturalmente, un objetivo así no es fácil de alcanzar cuando se pretende que además de la calidad brindada por los autores de los trabajos de investigación publicados, éstos sean de interés para quienes estudian el fenómeno de la administración de los diferentes tipos de organizaciones desde diferentes perspectivas teóricas y enfoques disciplinarios, así como para quienes ejercen en el campo de la administración para conducir al logro de sus objetivos a distintas organizaciones.

En un mundo dinámico, sujeto a la ley del cambio constante, es normal que encontremos nuevos desafíos para explicar y comprender mejor a las organizaciones y el entorno en que éstas actúan en pos de sus objetivos, haciendo que en ocasiones parezca que las perspectivas y enfoques con los que hemos tratado de hacer frente a esos desafíos que nos presenta la realidad no son ya suficientes. Por ello, como parte de la dinámica del mundo, tales perspectivas y enfoques se han enriquecido mediante nuevos planteamientos teóricos y herramentales analíticos, los cuales al sumarse al repertorio ya existente de explicaciones teóricas e instrumentos convencionales para el análisis contribuyen a enfrentar el estudio de las problemáticas administrativas con visiones enriquecidas.

Como editor de Contaduría y Administración es un placer privilegiado poner en manos de nuestros lectores esta entrega, el tercer número producido en su año 59 de vida, el cual es una muestra de ese dinamismo en el avance de los conocimientos que aportan diferentes disciplinas como contribuciones para incrementar la comprensión de las organizaciones actuales y su entorno. También esperamos que los adelantos que nuestros autores ponen ante nosotros fomenten los debates respectivos y permitan que se continúe avanzando en las pesquisas de nuestro interés.

Un asunto de interés relacionado con el empleo de los recursos humanos, obviamente además de la existencia de mano de obra suficiente en cantidad y calidad, es el propio contexto en que ésta se desenvuelve. En el caso de México y otros países de Latino América y del mundo existen problemas estructurales en relación con las dotaciones de mano de obra con las características deseables de cantidad y calidad dadas las 
necesidades que tienen las empresas privadas del sector productivo, situación que se empeora debido a que el funcionamiento de las estructuras económicas de varios de esos países se ha vuelto un expulsor sistemático de mano de obra, tanto calificada como no calificada, la cual tiende a desplazarse hacia economías con mayores niveles de actividad económica con la esperanza de encontrar un entorno propicio para continuar con su desarrollo personal, profesional y económico. Cuauhtémoc Calderón Villareal y Luis Huesca, en el trabajo titulado Profile of earners and remittances in Mexico: a relative deprivation approach, nos muestran los resultados de su estudio sobre características individuales y los perfiles microeconómicos de trabajadores mexicanos que deciden emigrar del país en la búsqueda de mejores condiciones de vida. Los autores de este trabajo encuentran que los programas de asistencia pública refuerzan la privación relativa de las familias en las cuales no hay migrantes en comparación con las familias en las cuales alguno(s) de sus miembros ha(n) migrado. En sentido contrario a como sería deseable esperar, ese tipo de programas actúa en realidad como un incentivo perverso en el sentido de que al aumentar la brecha entre los tipos de familias antes mencionados, para muchas personas se hace más deseable el salir del país a buscar un empleo que les permita mejorar sus condiciones de vida.

Además de los problemas relacionados con las disponibilidad de recursos humanos existen también problemas comunes de diversa índole que pueden incidir de manera decisiva en el desarrollo de las empresas, entre los cuales destaca el problema de la recaudación fiscal que enfrentan los diversos gobiernos del mundo para llevar a cabo sus labores como órgano administrativo del Estado y la inversión en infraestructura que proporcione mejores condiciones de vida a la población al mismo tiempo que eleva el potencial productivo de la nación mediante educación, salud y creación de infraestructura de comunicaciones y transporte. Gerardo Ángeles y Diana Berenice Ramírez nos presentan su artículo Determinants of tax revenue in OECD countries over the period 2001-2011, en el que estudian los impactos que tienen sobre los ingresos fiscales diferentes tipos de factores: económicos, estructurales, institucionales y sociales. El estudio abarca el análisis de esas relaciones en 34 países miembros de la Organización para la Cooperación Económica y el Desarrollo durante el periodo en cuestión. Entre los hallazgos de estos autores destaca que el PIB per cápita, el desempeño del sector industrial y las libertades civiles de las cuales goza el ciudadano son factores que tienen efectos positivos en los ingresos fiscales, en tanto que el sector agrícola y la participación de la inversión extranjera en la formación bruta de capital fijo tienen efectos negativos. También es conveniente señalar que su estudio muestra evidencia de que el ingreso fiscal rezagado ofrece una contribución importante al ingreso fiscal, particularmente en países con ingresos altos, es decir, cabe la posibilidad de que exista un círculo virtuoso en el que un buen nivel de ingreso fiscal coadyuva a mantener 
ingresos fiscales positivos con base en un mayor nivel de actividad empresarial y de la economía en general.

Con la evolución en la tecnología de las comunicaciones y de la información, quizá sería correcto decir revolución, se han presentado nuevos medios para la administración de la información clave para las actividades de las organizaciones. Debido a ese papel clave, la administración de los riesgos informáticos es una materia de importancia dentro de la emergente área de Administración de Riesgos Empresariales. María de los Ángeles López, Diana Ester Albanese y Marisa Analía Sánchez son autoras del trabajo Gestión de riesgos para la adopción de la computación en nube en entidades financieras de la República Argentina, en el cual se ocupan del estudio de los riesgos de la computación en nube en ese país. Sostienen que al analizar el uso de arquitecturas informáticas que hacen uso intensivo de información, como es el caso de las empresas del sector financiero, es de suma importancia tomar en cuenta los nuevos riesgos a que se exponen los diferentes usuarios. En lo particular, destacan la utilidad de la herramienta denominada risk breakdown structure (RBS) mediante la cual se pueden jerarquizar las fuentes de riesgo permitiendo simplificar y sistematizar el análisis de los riesgos. Proponen un diseño para una RBS apropiada a la computación en nube por parte de las entidades financieras argentinas con base en la normatividad que les impone el Banco Central de ese país.

En números previos de esta revista se han presentado investigaciones sobre diversos aspectos de la responsabilidad social, lo que naturalmente no implica que se hayan agotado en alguna forma los posibles debates en relación con ese tópico. Un cuestionamiento importante sobre la adopción de las prácticas de responsabilidad social es si tales prácticas se adoptan como un esfuerzo derivado de la conciencia social de las empresas o es única y exclusivamente una táctica de mercadotecnia para favorecer la imagen empresarial con el objetivo de aumentar las ventas y ganancias. Valentin Alejandro Martínez, Óscar Juanatey y Maria José da Silva, dan una muestra de lo vivo que se puede encontrar el interés por ese tema en su trabajo Impacto da responsabilidade social sob a óptica empresarial do norte litoral de Portugal. En su investigación, los autores tratan de verificar si las prácticas de responsabilidad social por parte de empresas ubicadas en la ciudad de Maia, en el norte de Portugal, tienen algún impacto en sus acciones y si se ve afectada positiva o negativamente la imagen corporativa de dichas empresas. El análisis empírico que llevan a cabo muestra que las prácticas de responsabilidad social si tienen impacto en las acciones de esas empresas, pero no encuentran efectos significativos en su desempeño económico. 
Otra discusión de interés muy actual es la relación entre el gobierno corporativo y el desempeño financiero de las empresas, siendo lo que cabe esperar que las prácticas de gobierno corporativo coadyuven a mejorar el desempeño empresarial debido a que la menor opacidad en la toma de decisiones disminuye el nivel de riesgo no sistemático que es percibido por los participantes de los mercados de la propiedad corporativa. El menor nivel de opacidad, gracias a la revelación de información importante sobre la empresa y sus acciones, mejora el proceso de valuación de los activos de la empresa debido a que ese mayor y mejor flujo de información ofrece un mejor sustento a la decisión de adquirir dichos activos al precio de mercado. En el artículo Governança corporativa: uma análise de sua relação com o desempenho econômico-financeiro de empresas cotadas no Brasil nos anos de 2010-2012, Anderson Catapan y Romualdo Douglas Colauto estudian la relación entre el gobierno corporativo y el desempeño financiero de las empresas brasileñas que cotizan en el mercado de valores brasileño. Encuentran una relación directa entre el valor de la empresa que percibe el mercado y el nivel de divulgación de información relevante, así como una relación entre el Índice de Gobierno Corporativo, la q de Tobin y el rendimiento de los activos. En resumen, la evidencia ofrecida en el trabajo de Catapan y Colauto muestra la importancia que tiene el gobierno corporativo para el desempeño financiero.

Entre los mecanismos de compensación laboral, el ahorro necesario para el retiro juega un papel muy importante pues se entiende que constituye un medio para compensar al trabajador por su participación en la generación de riqueza precisamente cuando más lo necesita: cuando ha terminado su vida activa desde el punto de vista económico. En el caso mexicano es conveniente recordar que ante una cantidad considerable de posiciones opuestas, quizá podría decirse incluso que a pesar de y por encima de ellas, se impuso a fines del siglo pasado el sistema de pensiones actualmente vigente bajo el argumento del gobierno de que ese era el único camino posible para que los trabajadores mexicanos contasen con un fondo que les proporcionara su pensión en el retiro. Por lo anterior, es de gran importancia estudiar ampliamente las características del riesgo al que se encuentran sujetos tales fondos, en el artículo Análisis del riesgo de mercado de los fondos de pensión en México: un enfoque con modelos autorregresivos Marissa Martínez y Francisco Venegas Martínez se ocupan del análisis del riesgo de dos de los principales tipos de fondos de pensión actualmente en operación, las denominadas SB1 y SB2, mediante un índice construido para medir su desempeño. El enfoque de modelado de econometría de series de tiempo con el que abordan su estudio, les lleva a concluir que los rendimientos obtenidos durante el periodo estudiado, no son suficientes para compensar el riesgo adicional que implica la inclusión de instrumentos de renta variable en los fondos de pensión. 
También en relación con los vínculos entre el rendimiento de activos financieros y su nivel de exposición al riesgo en México, en este número se presenta el artículo Estimation of the underlying structure of systematic risk with using principal component analysis and factor analysis, en el cual Rogelio Ladrón de Guevara y Salvador Torra-Porras muestran los resultados de su investigación sobre la estructura del riesgo sistemático en el mercado accionario mexicano. Partiendo de los fundamentos de la Teoría de Valuación por Arbitraje (APT, Arbitrage Pricing Theory), mediante la cual se intenta ofrecer una explicación al valor de los activos en el mercado con base en fuentes de riesgo sistemático, estos autores estudian dicha estructura bajo una perspectiva de factores estadísticos de riesgo extraídos de los rendimientos observados. Los tipos de rendimientos analizados son los rendimientos y los rendimientos en exceso de la tasa libre de riesgo, encontrando soporte parcial a los planteamientos de la APT.

El estudio de las respuestas de las empresas ante eventos desfavorables siempre es de gran importancia para comprender la forma en que se diseñan estrategias de sobrevivencia y el potencial de éxito y consecuencias de tales decisiones, las cuales no sólo afectan directamente a ciertos grupos de interés como son los propios empresarios, empleados y trabajadores, proveedores y clientes, por ejemplo, sino que también pueden extender los efectos negativos de esas decisiones, a manera de externalidades, hacia otras capas de la sociedad que no se encuentran directamente ligadas a la marcha de esas empresas. En el artículo Declive organizativo, fracaso y reestructuración organizacional en empresas colombianas, María Consuelo Mora analiza los procesos de reestructuración que siguieron empresas de Bogotá, Colombia, para enfrentar episodios de crisis económica entre 1995 y 2010. La profesora Mora destaca que las empresas estudiadas llevaron a cabo procesos de reestructuración con base en la reducción de su tamaño, los cuales les resultaron perjudiciales porque produjeron un acortamiento en los periodos de auge del ciclo económico. Una consecuencia inmediata de lo anterior, observa la autora, fue que se precipitó el cierre de empresas, en particular de tamaño grande y mediano, impactando negativamente a la sociedad debido a la ampliación de las brechas sociales y a la reducción consecuente en los niveles de vida.

Como lo señalan Sandra Alvear y José Tello en su artículo El discurso directivo en la comunicación de los estados financieros: el caso de dos empresas chilenas, el rol de portavoces de las empresas que es llevado a cabo por sus directivos es un objeto de estudio importante para quienes tratan de comprender mejor los procesos de dirección empresarial, siendo considerado el discurso de esos directivos un instrumento activo de su dirección. En este artículo se presentan trabajo los resultados de los esfuerzos hechos por Alvear y Tello para identificar, describir y comprender la función del discurso 
directivo mediante el estudio de la carta de presentación de los estados financieros de dos empresas colombianas. El análisis se llevo a cabo por medio de la identificación lexical de los enunciados, a partir de lo cual los autores infieren que en ambos casos los directivos intentan alinear los resultados económico-financieros de esas empresas con el contexto que enfrentan al mismo tiempo que tratan de influir en los miembros de ciertos grupos de presión social. También identifican dos dimensiones en el discurso directivo: una de carácter estratégico que se expresa como la imagen que la empresa desea difundir, la otra de carácter mercadotécnico mediante la cual la empresa intenta promoverse como un producto ante sus clientes, los accionistas.

Naturalmente, como lo han mostrado diferentes trabajos publicados, la comprensión de las relaciones entre las empresas que ofrecen sus productos, en forma de bienes y servicios, y los clientes, consumidores, son materia de estudio permanente. En el artículo Atributos relevantes de calidad en el servicio y su influencia hacia la lealtad de marca en la industria aseguradora de automóviles en México, Jorge Vera y Marco Tulio Espinosa nos presentan el estudio que realizaron con el objetivo de identificar cuáles son los atributos que conforman la calidad en el servicio en el mercado de seguros para automóvil y conocer cómo influyen en diversas mediciones de lealtad del cliente hacia la marca. En el diseño de su investigación, Vera y Espinosa establecen una distinción entre aspectos de atención al cliente durante la contratación y después del siniestro. Con base en el análisis de la información arrojada por las encuestas que realizan, pueden concluir sobre cuáles son los atributos de atención después del siniestro que tienen más influencia en la lealtad del cliente, encontrando que la reputación de la compañía aseguradora, atributo de contratación, también es importante para la lealtad.

Reiterando mi beneplácito, así como mis mejores deseos y agradecimiento a los lectores, autores, el equipo editorial de Contaduría y Administración y las personas que de una forma u otra nos apoyan dentro del marco permisible por sus posibilidades, me queda únicamente esperar que estos esfuerzos sean suficientemente satisfactorios y sirvan de acicate para seguir impulsando el desarrollo de esta revista.

Francisco López Herrera

Director Editorial 